\title{
Preoperative Grading of Astrocytic Supratentorial Brain Tumors with Diffusion-Weighted Magnetic Resonance Imaging and Apparent Diffusion Coefficient
}

\author{
Mahsa Raisi-Nafchi, ${ }^{1}$ Fariborz Faeghi, ${ }^{1,}$ Alireza Zali, ${ }^{2}$ Hamidreza Haghighatkhah, ${ }^{3}$ and Jalal \\ Jalal-Shokouhi $^{4}$ \\ ${ }^{1}$ Department of Radiology Technology, School of Allied Medical Sciences, Shahid Beheshti University of Medical Sciences, Tehran, Iran \\ ${ }^{2}$ Department of Neurosurgery, Functional Neurosurgery Research Center, Shohada Tajrish Hospital, Shahid Beheshti University of Medical Sciences, Tehran, Iran \\ ${ }^{3}$ Department of Radiology, Shohada Tajrish Hospital, Shahid Beheshti University of Medical Sciences, Tehran, Iran \\ ${ }^{4}$ Jam-e-Jam Medical Imaging Center, Tehran, Iran \\ "Corresponding author: Fariborz Faeghi, Department of Radiology Technology, School of Allied Medical Sciences, Shahid Beheshti University of Medical Sciences, Tehran, Iran, \\ E-mail: f_faeghi@sbmu.ac.ir
}

Received 2015 June 06; Revised 2015 October 14; Accepted 2015 October 20.

\begin{abstract}
Background: Diffusion-weighted imaging (DWI) is a form of magnetic resonance imaging (MRI) based on measuring the random Brownian motion of water molecules within the biological tissues and is particularly useful in tumor characterization.

Objectives: The purpose of this study was to evaluate the diagnostic value of DW MRI and the apparent diffusion coefficient (ADC) for preoperative grading of astrocytic supratentorial brain tumors.

Patients and Methods: Twenty-three patients (14 females, 9 males, mean age 43 years) with astrocytic supratentorial brain tumors underwent preoperative conventional MR imaging and DW MRI. The minimum, maximum and mean ADC values and the minimum, maximum and mean DWI signal intensities of each tumor were taken by placing several regions of interest in the tumor on DWI images and ADC maps. To assess the relationship between these values and the tumor grade, we used the Mann-Whitney U test and the Spearman correlation. Receiver operating characteristic (ROC) analysis was used to determine the cutoff value of the minimum, maximum and mean ADC values and the minimum, maximum and mean DWI signal intensities that had the best composition of sensitivity and specificity for differentiating low-grade and high-grade astrocytic brain tumors.

Results: According to the pathology reports, 10 patients had low-grade astrocytomas (grades I, II) and 13 patients had high-grade astrocytomas (grades III, IV). The minimum ADC value showed a significantly inverse correlation with astrocytic tumor grade $(\mathrm{P}=$ 0.006). The correlation between the maximum ADC value and the maximum DWI signal intensity with tumor grade was direct $(\mathrm{P}=$ $0.013, \mathrm{P}=0.035$ ). According to the ROC analysis, the cutoff values of $0.843 \times 10^{-3} \mathrm{~mm}^{2} / \mathrm{s}, 2.117 \times 10^{-3} \mathrm{~mm}^{2} / \mathrm{s}$ and 165.2 for the minimum $\mathrm{ADC}$, maximum ADC and maximum DWI respectively, obtained the best combination of sensitivity and specificity for distinguishing low-grade and high-grade astrocytomas.

Conclusion: Measuring minimum ADC, maximum ADC and maximum DWI signal intensity can provide valuable information for grading of astrocytic supratentorial brain tumors before surgery.
\end{abstract}

Keywords: Diffusion Magnetic Resonance Imaging, Diffusion Weighted MRI, Cerebral Astrocytoma, Neoplasm Grading, Tumor Grading

\section{Background}

Astrocytic brain tumors are derived from a particular type of glial cells, which are star-shaped brain cells in the central nervous system, called astrocytes. Astrocytoma does not usually spread outside the brain and this type of tumor may occur in most parts of the brain. Astrocytomas are the most common primary brain tumors and accounting for approximately $50 \%$ of all brain tumors and also accounting for more than $70 \%$ of glial brain tumors. According to the WHO (world health organization) classification, based on the degree of malignancy, astrocytomas can be divided into four grades. Astrocytomas grade I and II are called "low-grade astrocytomas," and astrocytomas grade III and IV are called "high-grade astrocytomas." They can occur at any age. The low-grade astrocytomas are more common in children, while the high-grade astrocytomas are more common in adults. Despite advanced treatments, the diagnosis and prognosis of brain tumors, especially astrocytic brain tumors is still poor. For this reason, even today, brain tumors are a common cause of morbidity and mortality. Hence, early diagnosis and awareness of preoperative tumor grade is important in choosing the appropriate treatment strategy and in increasing the patient's survival 
(1-5).

X-ray computed tomography (CT) and magnetic resonance imaging (MRI) are used for imaging of astrocytic brain tumors. Of these two methods, MRI has replaced CT as the modality of choice for evaluating these tumors. Nevertheless, only conventional magnetic resonance imaging techniques are not enough for awareness of the histopathologic grade of astrocytomas, because sometimes high-grade and low-grade astrocytic brain tumors have common features in conventional MR imaging (6). Recently, several techniques, in addition to conventional MR imaging, were examined for preoperative grading of brain tumors, such as diffusion-weighted magnetic resonance imaging (DWI) and apparent diffusion coefficient maps $(7,8)$. DWI is an MRI technique used to show the random Brownian motion of water molecules at the microscopic level within the biological tissues and the apparent diffusion coefficient (ADC) is a parameter that is calculated from the diffusion-weighted MR images (9-14). Also, recently, an inverse relationship was observed between the histopathologic grade of glial tumors and the minimum apparent diffusion coefficient (ADCmin) $(1,15)$.

\section{Objectives}

The purpose of this study was to obtain preoperative information about the grade of astrocytic supratentorial brain tumors with DWI and ADC maps with more detail and more accuracy than previous studies by measuring the minimum, maximum and mean ADC values and the minimum, maximum and mean DWI signal intensities.

\section{Patients and Methods}

\subsection{Patients}

Our prospective study included 23 patients (14 female, 9 male; mean age 43 years) with symptoms of brain tumors and in whom astrocytic supratentorial brain tumors were diagnosed after MR imaging and review by a neurosurgeon. All patients underwent conventional MR imaging techniques and diffusion-weighted MR imaging before treatment or surgery. In addition, all histopathologic diagnosis of tumor grade were determined on the basis of the WHO criteria. So that, among 23 patients, 10 patients had low-grade astrocytomas (I, II) and 13 patients had highgrade astrocytomas (III, IV). It should be noted that informed consent was obtained from all patients and all ethical considerations were considered in this study.

\subsection{MR Imaging and Image Processing}

All MR examinations were performed with a 1.5 T MR imaging system (Siemens medical systems, Avanto, Germany) with a standard eight-channel head coil. First, for determining the exact location of the lesion, conventional MR imaging protocols included fluid attenuated inversion recovery (FLAIR), T1 weighted spin-echo ( $\mathrm{T}_{1} \mathrm{~W}$ SE), T2 weighted fast spin-echo ( $\mathrm{T}_{2} \mathrm{~W}$ FSE) and post-contrast $\mathrm{T}_{1} \mathrm{~W}$ SE images were taken. Then, the DWI protocol was performed by using a spin-echo echo-planar sequence with 0 and $1000 \mathrm{~b}$-values and by applying the diffusion gradients encoding in three orthogonal directions ( $\mathrm{TR}=3700 \mathrm{~ms} / \mathrm{TE}$ $=114 \mathrm{~ms} /$ number of signals acquired $=1 /$ slice thickness $=5$ $\mathrm{mm} /$ interslice gap $=0 /$ matrix $=192 \times 192 / \mathrm{FOV}=240 \times 240$ $\mathrm{mm})$. ADC maps were calculated as well. Next, the minimum, maximum and mean ADC values and the minimum, maximum and mean DWI signal intensities of each tumor were taken by placing several ROIs (Regions of Interest) in the tumor on DWI images and ADC maps (Figure 1). All continuous slices of ADC maps and DWI images that included astrocytic tumors were checked. The number of ROIs, according to the tumor size, was selected and their numbers were selected for covering the entire geographical area of tumor. In all patients, the area of each ROI was $33 \mathrm{~mm}^{2}$ and contained 21 pixels. On ADC maps and DWI images, among all regions of interest, ROIs with the lowest and highest values as the minimum and maximum amount of ADC values and the minimum and maximum amount of DWI signal intensities were chosen. Then, for obtaining the mean ADC values and the mean DWI signal intensities between the maximum and minimum values were averaged.

\subsection{Statistical Analysis}

To analyze the minimum, maximum and mean ADC values and the minimum, maximum and mean DWI signal intensities of various astrocytic brain tumors, we subdivided these tumors into two groups: low-grade astrocytomas (I, II) as group one and high-grade astrocytomas (III, IV) as group two.

To assess the relationship between these values and tumor grade, and also the relationship between the age of the patient and tumor grade, we used the Mann-Whitney $\mathrm{U}$ test and spearman correlation coefficient. ROC (receiver operating characteristic) analysis was used to define the cutoff value of the minimum, maximum and mean ADC values and the minimum, maximum and mean DWI signal intensities that had the best combination of sensitivity and specificity for distinguishing low-grade and high-grade astrocytic brain tumors. 

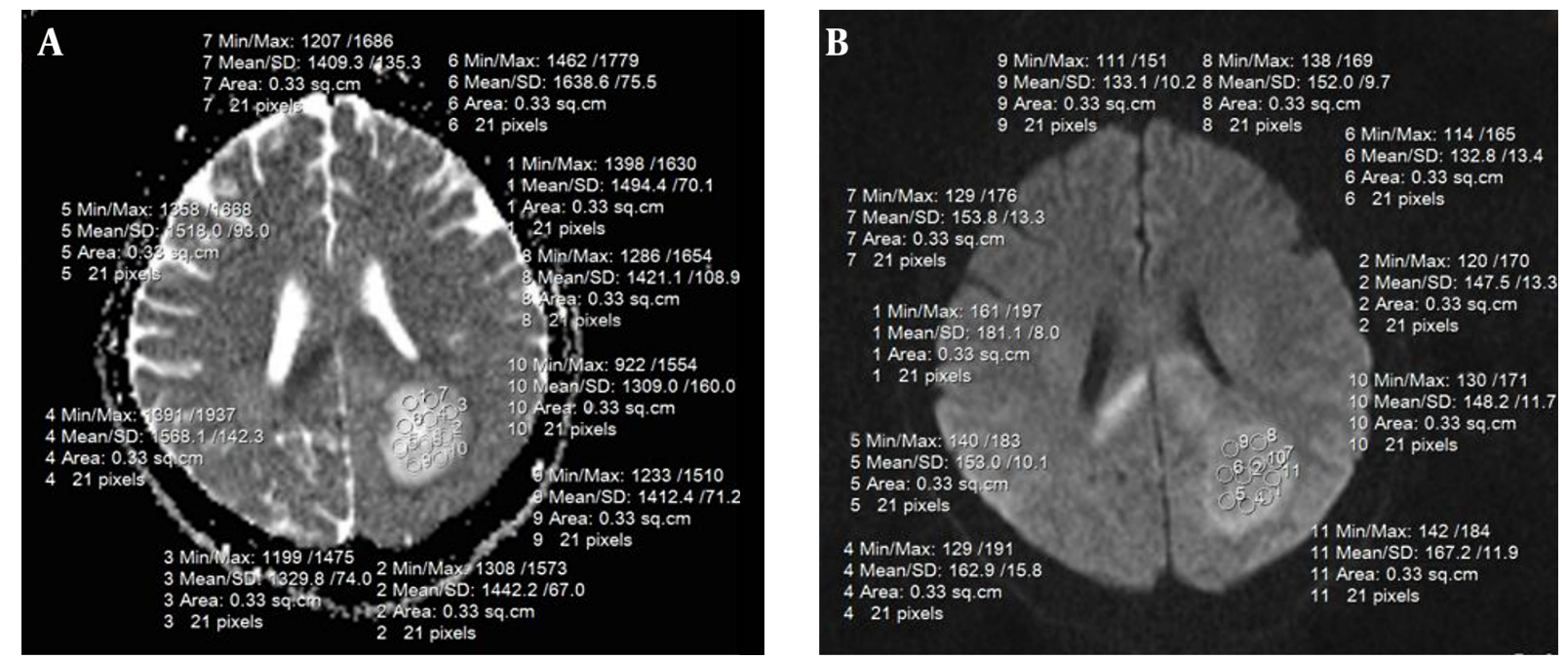

Figure 1. A 43-year-old woman with glioblastoma multiforme; $A, A D C$ map; B, DWI image. ROIs drawn on the tumor, as well as, ADC values and DWI signal intensities obtained from each ROI can be seen in the image.

\section{Results}

Among 23 patients who had histologically confirmed their astrocytomas, there were three patients with astrocytoma grade I, seven patients with astrocytoma grade II, five patients with astrocytoma grade III and eight patients with astrocytoma grade IV (Table 1).

The relationship between the minimum ADC values and tumors grade were significantly reversed $(\mathrm{P}=0.006)$ and the correlation coefficient value between ADCmin and tumor grade was "-0.582" (Figure 2, Table 2). The maximum ADC values and maximum DWI signal intensities also were significantly direct correlation with grade of tumors $(\mathrm{P}=$ 0.013 and $\mathrm{P}=0.035$, respectively), and the correlation coefficient value between ADCmax and the tumor grade was 0.529 . In addition, the correlation coefficient value between DWImax and the tumor grade was 0.450 (Figure 3, Table 2).

According to the ROC analysis, the cutoff value of 0.843 $\times 10^{-3} \mathrm{~mm}^{2} / \mathrm{s}$ for ADCmin with $80 \%$ sensitivity and $84.6 \%$ specificity (area under the curve $[$ AUC $]=0.838$, positive predictive value $[\mathrm{PPV}]=84.6 \%$, negative predictive value $[\mathrm{NPV}]$ $=80 \%$ ), the cutoff value of $2.117 \times 10^{-3} \mathrm{~mm}^{2} / \mathrm{s}$ for ADCmax with $76.9 \%$ sensitivity and $90 \%$ specificity $($ AUC $=0.808, \mathrm{PPV}$ $=90 \%, \mathrm{NPV}=75 \%)$ and the cutoff value of 165.2 for DWImax with $84.6 \%$ sensitivity and $70 \%$ specificity $(A U C=0.762$, PPV $=78.5 \%, \mathrm{NPV}=77.7 \%)$ were chosen for differentiating the low-grade and high-grade astrocytomas (Figure 4). Also, no statistically significant correlation between tumor grade with ADCmean, DWImin, DWImean and age of patient was observed $(\mathrm{P}=0.137, \mathrm{P}=0.193, \mathrm{P}=0.094$ and $\mathrm{P}=0.062$, respec-

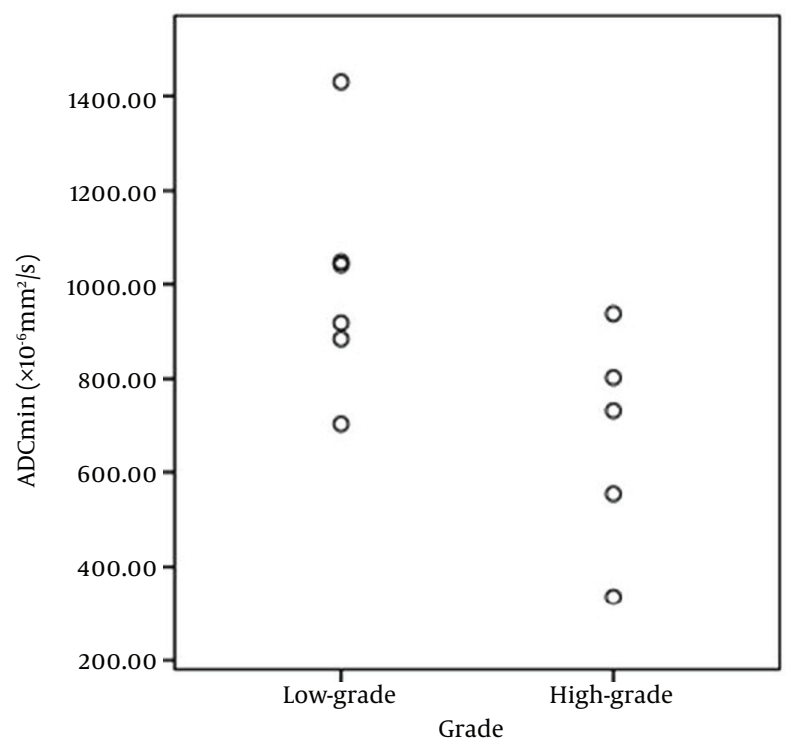

Figure 2. The correlation between ADCmin and grade of astrocytomas; minimum $\mathrm{ADC}$ values of high-grade astrocytomas are lower than ADC values of low-grade astrocytomas, however, some overlap is noticed between the two groups.

tively).

\section{Discussion}

As stated, using only conventional MRI may not always be reliable for predicting the histopathologic grading of 

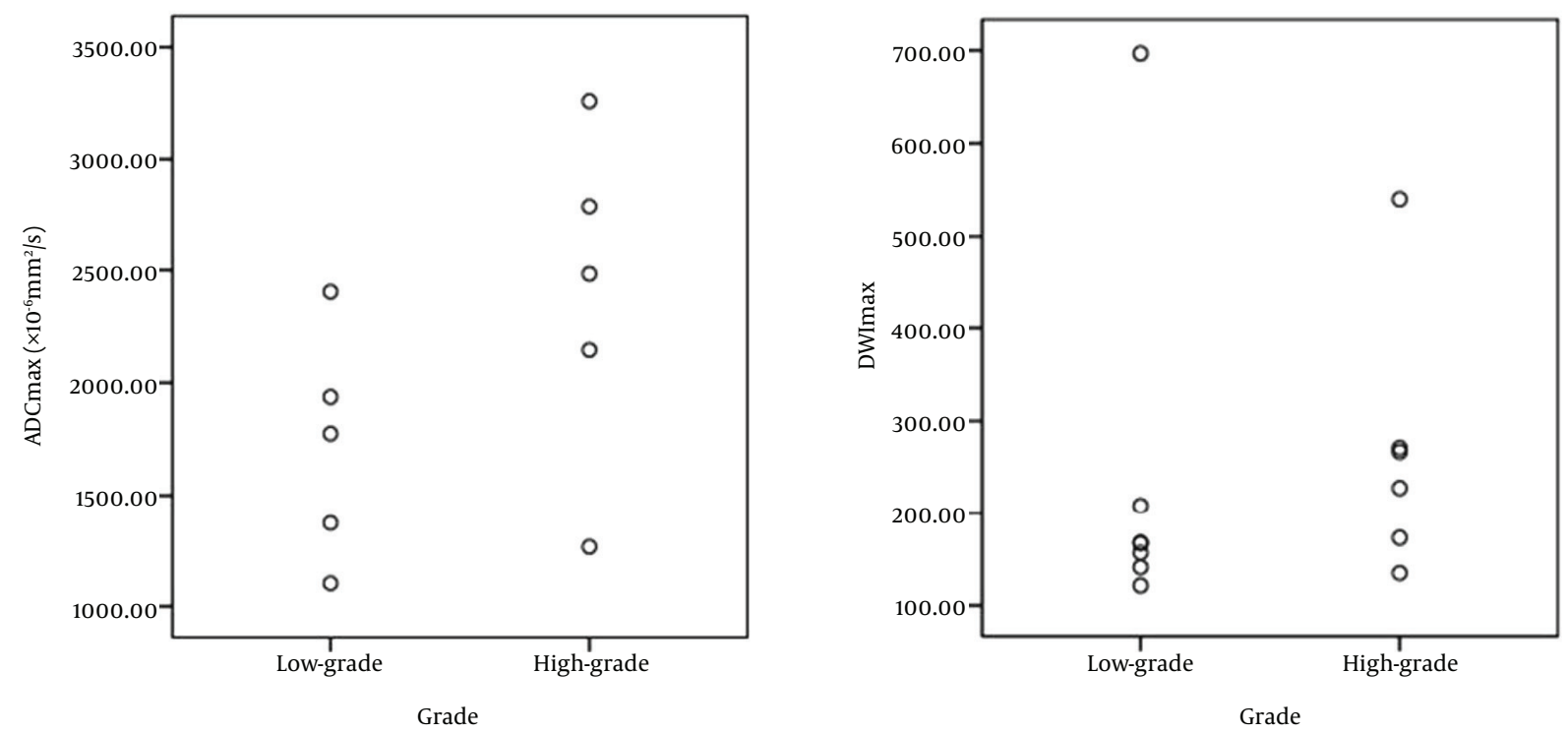

Figure 3. The correlation between ADCmax and DWImax with the grade of astrocytomas; maximum ADC values and maximum DWI signal intensities of high-grade astrocytomas are higher than those of low-grade astrocytomas, however, some overlap is noticed between the two groups.

the astrocytic supratentorial brain tumors, because the different grades of these tumors can have common features in conventional MRI. In recent years, many researchers have studied the diagnostic value of DWI and the ADC for the diagnosis of brain lesions, and they have declared that the capability of MRI to measure and imaging of molecular diffusion, can produce more accurate information about the brain lesions than conventional MR imaging $(16,17)$. Knowing the grade of brain tumors is very important for choosing the appropriate treatment strategy. DWI over conventional MRI allows the evaluation of brain tumors by providing information about tumor cellularity, which can improve the prediction of tumor grade $(13,18)$. Some studies have reported a correlation between tumor cellularity and ADC values, so that lower ADC values show highertumor grades. These studies have stated that ADC can be useful in determining the grade of gliomas, because highgrade gliomas have high cellularity and lower ADC values compared to the low-grade gliomas. For example, in 2001, Kono et al. evaluated the role of DWI in patients with brain tumors. They examined 56 patients with histologically verified or clinically diagnosed brain tumors. Then, they measured ADC values and signal intensities on DWIs and evaluated the correlation between ADC values and tumor cellularity in gliomas. They found that ADC values of lowgrade astrocytomas are significantly higher than those of other tumors and among astrocytic brain tumors; ADCs are higher in grade II astrocytomas than in gliobastomas.
In addition, the ADC values correlated with tumor cellularity for astrocytic tumors. In their research, they concluded that ADC may predict the degree of malignancy of astrocytic tumors (19).

Also, in 2005 Yamasaki et al. performed a study to determine the role of ADC in differentiating brain tumors by MRI. In their retrospective study, 275 patients with brain tumors were examined after providing DWI images and calculating the mean ADC values and analyzing these values. They concluded that an inverse relationship existed between the mean ADC values and the grade of astrocytic brain tumors (grade II to IV) $(13,20)$. In 2006, Fan et al. conducted a study in order to evaluate the usefulness of diffusion and perfusion MR imaging in patients with nonenhancing supratentorial brain gliomas. They examined 22 patients; 14 had low-grade gliomas and 8 had anaplastic gliomas. They obtained the ADC values and relative cerebral blood volume ( $\mathrm{rCBV}$ ) ratios on the solid portion of the tumor, on the peritumoral area, as well as on the contralateral normal white matter. After data analysis, they declared that DWI should be used in the diagnostic workup of nonenhancing gliomas to predict grading (21). However, some older studies are inconsistent, for example, in 1999, Sugahara et al. performed a study to evaluate the utility of DWI with the echo-planar imaging (EPI) technique in depicting tumor cellularity and grading of gliomas. They examined 20 patients with histologically proven gliomas. They also measured the cellularity of tumors and the minimum ADC 
A

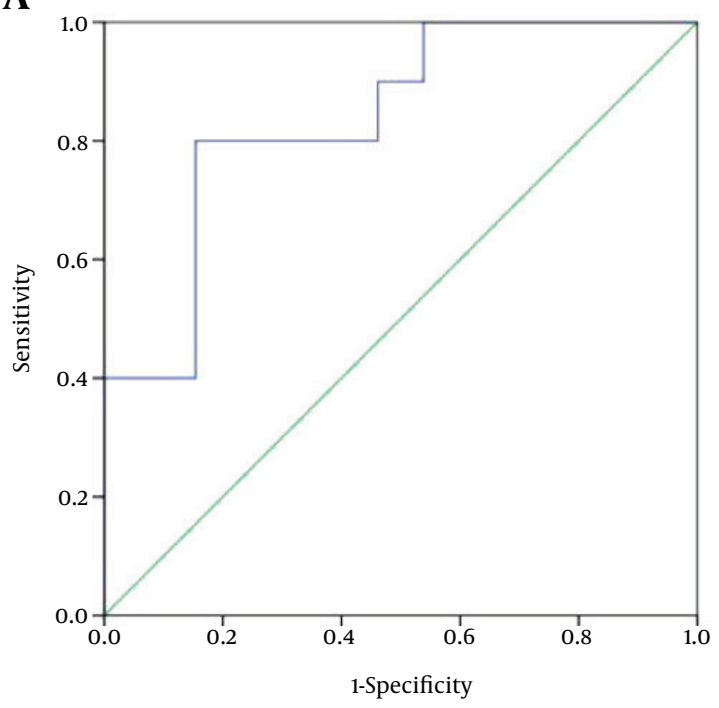

B

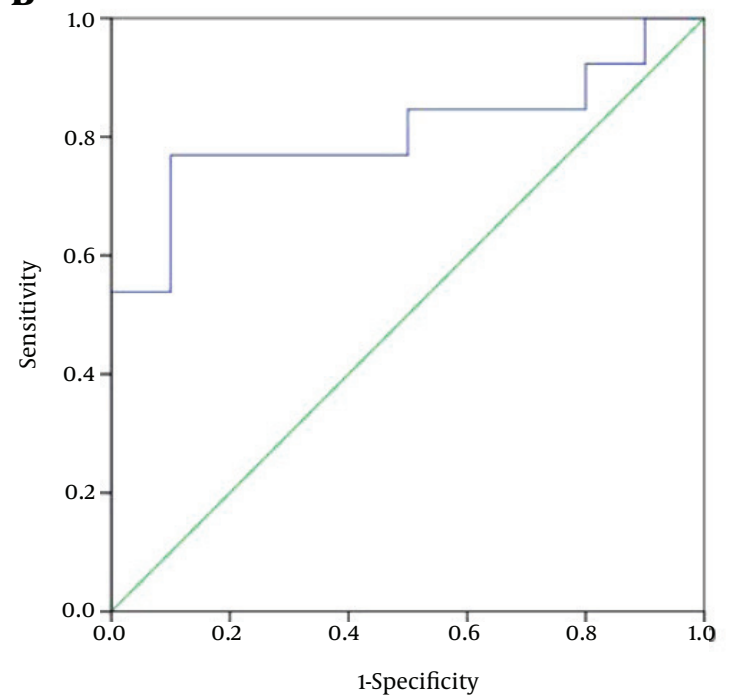

C

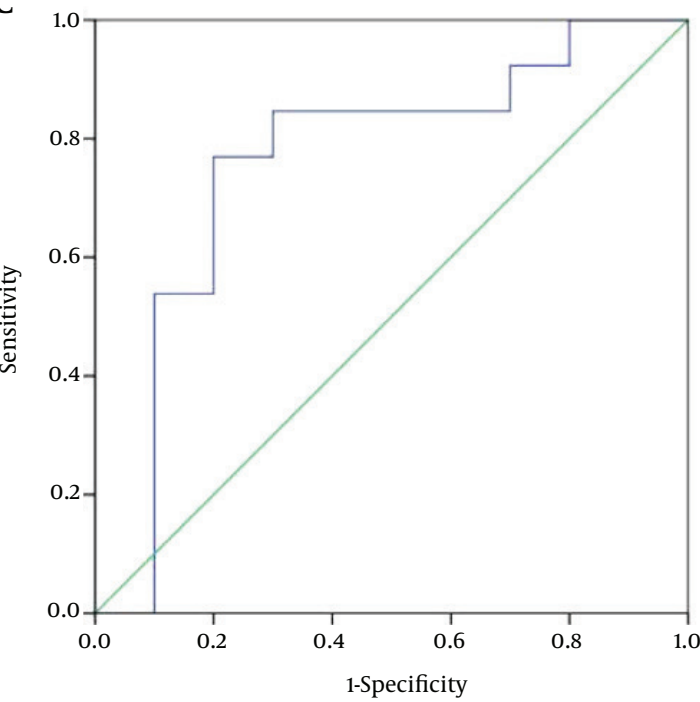

Figure 4. A, ROC curve of the ADCmin values; B, ROC curve of the ADCmax values; and C, ROC curve of the DWImax values.

values. Their results showed a relationship between the tumor cellularity and the minimum ADC value of gliomas, so that the minimum ADC value of the high-grade gliomas was higher than that of the low-grade gliomas. Finally, they reported that diffusion-weighted MRI with EPI is a useful technique for assessing the tumor cellularity and grading of gliomas (22).

However, there are some studies that have not confirmed this and have stated that no significant relationship exists between the ADC values and the grade of the gliomas (18). For example, in 2001 Lam et al. evaluated the role of DWI for grading gliomas in 17 patients. After providing the DWI images and ADC maps, as well as measuring the ADC values, they concluded that there is no significant difference between the ADC values of high-grade gliomas and low-grade gliomas (23). Also in 2006, Rollin et al. studied the role of diffusion MR imaging for evaluation of grade and type of intra-axial brain tumors. They measured the ADC values in 28 patients and after analyzing these values they concluded that differentiating between low-grade and high-grade gliomas only by DWI images and ADC values is not possible (24). In other stud- 
Table 1. Gender, Age and Tumor Grade of Patients

\begin{tabular}{|c|c|c|c|}
\hline Patient & Gender & Age, $y$ & Grade \\
\hline $\mathbf{1}$ & M & 8 & Grade I \\
\hline 2 & $\mathrm{~F}$ & 11 & Grade I \\
\hline 3 & $\mathrm{~F}$ & 9 & Grade I \\
\hline 4 & M & 49 & Grade II \\
\hline 5 & $\mathrm{~F}$ & 45 & Grade II \\
\hline 6 & M & 48 & Grade II \\
\hline 7 & $\mathrm{~F}$ & 33 & Grade II \\
\hline 8 & M & 52 & Grade II \\
\hline 9 & $\mathrm{~F}$ & 29 & Grade II \\
\hline 10 & $\mathrm{~F}$ & 58 & Grade II \\
\hline 11 & M & 51 & Grade III \\
\hline 12 & F & 72 & Grade III \\
\hline 13 & $\mathrm{~F}$ & 20 & Grade III \\
\hline 14 & M & 39 & Grade III \\
\hline 15 & $\mathrm{~F}$ & 39 & Grade III \\
\hline 16 & $\mathrm{~F}$ & 52 & Grade IV \\
\hline 17 & $\mathrm{~F}$ & 43 & Grade IV \\
\hline 18 & M & 32 & Grade IV \\
\hline 19 & M & 71 & Grade IV \\
\hline 20 & $\mathrm{~F}$ & 52 & Grade IV \\
\hline 21 & M & 65 & Grade IV \\
\hline 22 & F & 63 & Grade IV \\
\hline 23 & F & 51 & Grade IV \\
\hline
\end{tabular}

Abbreviations: F, female; M, male.

ies only minimum ADC values have been evaluated, which were obtained from tumors. For example, in 2005 Higano et al. evaluated the ADC for prediction of malignancy and prognosis of malignant astrocytic brain tumors. They examined 37 patients with malignant astrocytomas that included 22 glioblastomas and 15 anaplastic astrocytomas. Then, they calculated ADC maps and measured the minimum ADC values of each tumor, preferably with the avoidance of cystic or necrotic parts. Eventually, they announced that minimum ADC values, which were obtained from malignant astrocytomas, can provide valuable information about their malignancy (15). Also, in 2006 Murakami et al. examined the diagnostic value of pretreatment diffusionweighted MR imaging in patients with malignant supratentorial astrocytic brain tumors. They evaluated the minimum $\mathrm{ADC}$ values as a factor analysis of survival in 79 patients. Of their patients, 29 had anaplastic astrocytoma and 50 had glioblastoma multiforme. After statistical anal- ysis, they found that the minimum ADC values were significantly lower in patients with glioblastoma multiforme than in those with anaplastic astrocytoma. Therefore, they concluded that MR imaging is a useful prognostic factor for survival in patients with malignant supratentorial astrocytomas. Our study confirms this (25).

Also, in 2008 Lee et al. assessed the diagnostic value of minimum ADC value for preoperative grading of supratentorial astrocytomas. In that study, they evaluated $16 \mathrm{pa}-$ tients with astrocytoma and calculated the minimum ADC value of each tumor from several regions of interest in the tumor on ADC maps. After data analysis, they reported that measuring the $\mathrm{ADCmin}$ can provide valuable information for preoperative grading of low-grade supratentorial astrocytomas, which is compatible with our results (1).

In the current study, we examined only astrocytic supratentorial brain tumors among all brain tumors, and in addition to minimum $A D C$ values we measured the maximum and mean $A D C$ values and the minimum, maximum and mean DWI signal intensities. After statistical analysis we concluded that the minimum ADC values had an inverse correlation with the tumor grade, and that the correlation between the maximum ADC values and maximum DWI signal intensities with tumor grade was direct, however, there is a slight overlap between the values of the two groups. The cutoff values of $0.843 \times 10^{-3} \mathrm{~mm}^{2} / \mathrm{s}, 2.117 \times 10^{-3}$ $\mathrm{mm}^{2} / \mathrm{s}$ and 165.2 were selected, in order to achieve ADCmin, ADCmax and DWImax with the best combination of sensitivity and specificity for differentiating high-grade and low-grade astrocytomas. The results showed that our study was much more accurate than previous studies.

There were two limitations in our study. First, was the small sample size and the other was that the assessment of reliability was not performed.

In conclusion, conventional MRI findings of astrocytic brain tumors is not always reliable for grading tumors. In this study, we showed that an inverse correlation exists between the minimum ADC values and histopathologic grade of the astrocytic supratentorial brain tumors. In addition, we showed a direct correlation between the maximum $A D C$ values and also the maximum DWI signal intensities with the grade of these tumors. Finally, we believe that measuring these values can provide valuable information for surgeons about the preoperative grading of astrocytic supratentorial brain tumors. 
Table 2. Mean of ADC and DWI Values in Low and High-Grade Tumors and Their Correlation Coefficient with Tumor Grade

\begin{tabular}{lcccc}
\hline Variables & Low-Grade $^{\mathbf{a}}$ & High-Grade $^{\mathbf{a}}$ & PValue $^{\mathbf{b}}$ & Correlation Coefficient \\
\hline ADCmin & $960.5(63.88)$ & $672.5(54.12)$ & 0.006 & -0.582 \\
ADCmax & $1731.9(122.18)$ & $2390.1(180)$ & 0.013 & 0.529 \\
DWImax & $209.65(54.61)$ & $237.84(28.75)$ & 0.035 & 0.450 \\
\hline
\end{tabular}

Abbreviations: ADC, apparent diffusion coefficient; min, minimum; max, maximum; DWI, diffusion weighted imaging; SD, standard deviation.

${ }^{a}$ Values are expressed as mean (SD).

${ }^{\mathrm{b}} \mathrm{P}$ Value for comparing mean values in low and high-grade tumors.

\section{Footnote}

Authors' Contribution: Study concept and design: Mahsa Raisi-Nafchi, Fariborz Faeghi, Alireza Zali, and Hamidreza Haghighatkhah; acquisition of data: Mahsa Raisi-Nafchi, Alireza Zali, and Hamidreza Haghighatkhah; analysis and interpretation of data: Mahsa Raisi-Nafchi, Fariborz Faeghi, Alireza Zali, and Hamidreza Haghighatkhah; drafting of the manuscript: Mahsa Raisi-Nafchi; critical revision of the manuscript for important intellectual content: Alireza Zali and Hamidreza Haghighatkhah; statistical analysis: Mahsa Raisi-Nafchi and Fariborz Faeghi; administrative, technical, and material support: Fariborz Faeghi, Alireza Zali, Hamidreza Haghighatkhah, and Jalal Jalal-Shokouhi; study supervision: Fariborz Faeghi, Alireza Zali, Hamidreza Haghighatkhah, and Jalal Jalal-Shokouhi.

\section{References}

1. Lee EJ, Lee SK, Agid R, Bae JM, Keller A, Terbrugge K. Preoperative grading of presumptive low-grade astrocytomas on MR imaging: diagnostic value of minimum apparent diffusion coefficient. AJNR Am J Neuroradiol. 2008;29(10):1872-7. doi: 10.3174/ajnr.A1254. [PubMed: 18719036].

2. Newton HB, Jolesz FA. Low-grade astrocytomas: Handbook of neurooncology neuroimaging. United States of America: Academic Press; 2007.

3. Wang YW, Yin CL, Zhang HY, Hao J, Yang YY, Liao H, et al. High expression of forkhead box protein C2 is related to poor prognosis in human gliomas. Asian Pac J Cancer Prev. 2014;15(24):10621-5. [PubMed: 25605149].

4. Newton HB, Jolesz FA. malignant astrocytomas: Handbook of neurooncology neuroimaging. USA: Academic Press; 2007.

5. Muallaoglu S, Besen AA, Ata A, Mertsoylu H, Arican A, Kayaselcuk F, et al. Lack of prognostic significance of C-erbB-2 expression in low- and high- grade astrocytomas. Asian Pac J Cancer Prev. 2014;15(3):1333-7. [PubMed: 24606461].

6. Drevelegas A. imaging modalities in brain tumors: Imaging of brain tumors with histological correlations. Philadelphia: Springer; 2010.

7. Svolos P, Tsolaki E, Kapsalaki E, Theodorou K, Fountas K, Fezoulidis $\mathrm{I}$, et al. Investigating brain tumor differentiation with diffusion and perfusion metrics at 3T MRI using pattern recognition techniques. Magn Reson Imaging. 2013;31(9):1567-77. doi:10.1016/j.mri.2013.06.010. [PubMed: 23906533].

8. Hygino da Cruz LC Jr., Vieira IG, Domingues RC. Diffusion MR imaging: an important tool in the assessment of brain tumors. Neuroimag- ing Clin N Am. 2011;21(1):27-49. doi: 10.1016/j.nic.2011.01.010. [PubMed: 21477750] vii.

9. Zhang H, Ma L, Shu C, Wang YB, Dong LQ. Diagnostic accuracy of diffusion MRI with quantitative ADC measurements in differentiating glioma recurrence from radiation necrosis. J Neurol Sci. 2015;351(12):65-71. doi: 10.1016/j.jns.2015.02.038. [PubMed: 25748965].

10. Doganay S, Kocakoc E, Cicekci M, Aglamis S, Akpolat N, Orhan I. Ability and utility of diffusion-weighted MRI with different b values in the evaluation of benign and malignant renal lesions. Clin Radiol. 2011;66(5):420-5. doi: 10.1016/j.crad.2010.11.013. [PubMed: 21334604].

11. Kızılgoz V. , Aydin H. , Hekimoglu B. . The efficacy of diffusion weighted imaging and apparent diffusion coefficient mapping for meniscal tears in the knee. Sci J Clin Med. 2013;2(6):171-5. doi: 10.11648/j.sjcm.20130206.15.

12. Wang Y, Lv X, Gong H, Yuan G, Zhang B, Zhao H, et al. Acute irradiation injury of canine brain with pathology control is detected by diffusion-weighted imaging of MRI. Clin Imaging. 2013;37(3):440-5. doi: 10.1016/j.clinimag.2012.09.006. [PubMed: 23102926].

13. Karaarslan E, Arslan A. Diffusion weighted MR imaging in noninfarct lesions of the brain. Eur J Radiol. 2008;65(3):402-16. doi: 10.1016/j.ejrad.2007.04.023. [PubMed: 17555903].

14. Young GS. Advanced MRI of adult brain tumors. Neurol Clin. 2007;25(4):947-73. doi: 10.1016/j.ncl.2007.07.010. [PubMed: 17964022] viii.

15. Higano S, Yun X, Kumabe T, Watanabe M, Mugikura S, Umetsu A, et al. Malignant astrocytic tumors: clinical importance of apparent diffusion coefficient in prediction of grade and prognosis. $\mathrm{Ra}$ diology. 2006;241(3):839-46. doi: 10.1148/radiol.2413051276. [PubMed: 17032910].

16. Newton HB, Jolesz FA. Diffusion imaging of brain tumors :Handbook of neuro-oncology neuroimaging. USA: Academic Press; 2007.

17. Kitis O, Altay H, Calli C, Yunten N, Akalin T, Yurtseven T. Minimum apparent diffusion coefficients in the evaluation of brain tumors. Eur J Radiol. 2005;55(3):393-400. doi: 10.1016/j.ejrad.2005.02.004. [PubMed: 16129247].

18. Newton HB, Jolesz FA. Diffusion magnetic resonance imaging in brain tumors: Handbook of neuro-oncology neuroimaging. USA:Academic Press; .

19. Kono K, Inoue Y, Nakayama K, Shakudo M, Morino M, Ohata K, et al. The role of diffusion-weighted imaging in patients with brain tumors. AJNR Am J Neuroradiol. 2001;22(6):1081-8. [PubMed: 11415902].

20. Yamasaki F, Kurisu K, Satoh K, Arita K, Sugiyama K, Ohtaki M, et al. Apparent diffusion coefficient of human brain tumors at MR imaging. Radiology. 2005;235(3):985-91. doi: 10.1148/radiol.2353031338. [PubMed: 15833979].

21. Fan GG, Deng QL, Wu ZH, Guo QY. Usefulness of diffusion/perfusionweighted MRI in patients with non-enhancing supratentorial brain gliomas: a valuable tool to predict tumour grading?. Brit J Radiol. 2006;79(944):652-8. doi: 10.1259/bjr/25349497.

22. Sugahara T, Korogi Y, Kochi M, Ikushima I, Shigematu Y, Hirai T, et al. Usefulness of diffusion-weighted MRI with echo-planar technique 
in the evaluation of cellularity in gliomas. J Magn Reson Imaging. 1999;9(1):53-60. [PubMed: 10030650].

23. Lam WW, Poon WS, Metreweli C. Diffusion MR imaging in glioma: does it have any role in the pre-operation determination of grading of glioma?. Clin Radiol. 2002;57(3):219-25. doi:10.1053/crad.2001.0741. [PubMed: 11952318].

24. Rollin N, Guyotat J, Streichenberger N, Honnorat J, Tran Minh VA, Cotton F. Clinical relevance of diffusion and perfusion magnetic resonance imaging in assessing intra-axial brain tumors. Neuroradiology. 2006;48(3):150-9. doi: 10.1007/s00234-005-0030-7. [PubMed: 16470375].

25. Murakami R, Sugahara T, Nakamura H, Hirai T, Kitajima M, Hayashida $\mathrm{Y}$, et al. Malignant supratentorial astrocytoma treated with postoperative radiation therapy: prognostic value of pretreatment quantitative diffusion-weighted MR imaging. Radiology. 2007;243(2):493-9. doi: 10.1148/radiol.2432060450. [PubMed: 17356177]. 